\title{
Estimation of the ground state correlation energy for isoelectronic series of 2 to 20 electrons *
}

\author{
K. Rashid ${ }^{1}$, B. Fricke ${ }^{2}$, D. Heinemann ${ }^{2}$, and Z.A. Saadi ${ }^{3}$ \\ 1 Centre of Basic Sciences, University Grants Commision, Islamabad, Pakistan \\ ${ }^{2}$ Department of Physics, University of Kassel, D-3500 Kassel, \\ Federal Republic of Germany \\ ${ }^{3}$ Pakistan Institute of Nuclear Science and Technology P.O. Nilore, Islamabad, \\ Pakistan
}

Received 12 June, 1987

\begin{abstract}
Correlation energies for all isoelectronic sequences of 2 to 20 electrons and $Z=2$ to 25 are obtained by taking differences between theoretical total energies of Dirac-Fock calculations and experimental total energies. These are pure relativistic correlation energies because relativistic and QED effects are already taken care of. The theoretical as well as the experimental values are analysed critically in order to get values as accurate as possible. The correlation energies obtained show an essentially consistent behaviour from $Z=2$ to 17 . For $Z>17$ inconsistencies occur indicating errors in the experimental values which become very large for $Z>25$.
\end{abstract}

PACS: $31.20 . \mathrm{Ej} ; 31.20 . \mathrm{Tz}$

\section{Introduction}

The recent decade has seen not only a revival of interest but also a significant increase of the pace of atomic physics research, for both theory and experiment. For theory the availability of high speed computers enabled tedious and sophisticated calculations of complex many-electron atomic systems. A number of programs for atomic structure calculations are now available [1-3]. On the experimental side large advances were made in collecting extensive experimental data, ranging from high Rydberg states to highly charged ions. Some of the most exciting results are recent measurements of hydrogen- and helium-like elements up to Uranium [4].

The most important quantity which characterises a static quantum mechanical system is the total energy $E$ which appears directly in the Schrödinger or Dirac equation. For many-electron systems the exact solution of this problem - non-relativistic or relativis-

* Dedicated to Professor Siegfried Penselin on the occasion of his 60th birthday tic - is not possible. In general, a large number of approximations must be introduced to obtain the solution. The approximation most widely used in calculating the details of atomic structure is the self-consistent field Hartree-Fock method [5]. For atomic systems containing many electrons this seems to provide the only practical recipe for their general solution. Since its inception it has evolved into a comprehensive working model for ab initio calculations of properties of many-electron atoms. One objective of this work is to calculate the total energies of ions and atoms from $Z=2-25$ by means of the fully relativistic version of the self consistent Hartree-Fock procedure (usually called Dirac-Fock) with the inclusion of the contributions of the quantum electrodynamical effects, vacuum polarisation and self-energy, as well as those of the Breit Hamiltonian in the electron-electron interaction. All these additional effects are calculated in first order perturbation theory following the $\mathrm{SCF}$ procedure. In addition the effect of the extended nucleus is taken into account by using a square well nuclear charge distribution with the nuclear parameters introduced in Ref. 6. 
Another objective is a systematic comparison of theoretical and experimental values to obtain insight into the accuracy of both. The difference between the relativistic Dirac-Fock total energies and the observed experimental values provides us with the correlation energy. We discuss this quantity as function of the number of electrons and the nuclear charge $Z$. Whenever possible, a comparison is made with other calculations, in particular with those of Clementi $[7]$.

\section{The total energy}

The total energy of a quantum mechanical system appears in the stationary state-equation as its eigenvalue

$H \Psi=E \Psi$,

$H$ being the Hamiltonian of the system, and $\Psi$ the total wave function which describes the state of the system. In relativistic quantum theory the total Hamiltonian $H$ of a many-electron atom is given by

$H=\sum_{i} h_{D}(i)+\sum_{i, j} g(i, j)$,

where $h_{D}(i)$ is the one-electron Dirac operator of the $i$ 'th electron

$h_{D}(i)=c \boldsymbol{\alpha}_{i} \cdot \mathbf{p}_{i}+\beta_{i} m c^{2}+V_{i}^{N}(r)$.

$c$ is the velocity of light, $\mathbf{p}_{i}$ the momentum of the $i$ 'th electron, $\alpha$ and $\beta$ are the $4 \times 4$ Dirac matrices usually used, and $V_{i}^{N}(r)$ is the electron-nucleus interaction energy. $g(i, j)$ is the two-electron operator representing the electron-electron interaction between $i$ 'th and $j$ th electron. In relativistic quantum theory this operator consists of the Coulomb term and an additional Breit term $[8] H_{B}(i, j)$

$g(i, j)=1 / r_{i j}+H_{B}(i, j)$.

The Breit term $H_{B}(i, j)$ is the sum of the magnetic and retardation interactions

$H_{B}(i, j)=-\frac{1}{2 r_{i j}}\left\{\left(\boldsymbol{\alpha}_{i}, \boldsymbol{\alpha}_{j}\right)+\frac{\left(\boldsymbol{\alpha}_{i}, \mathbf{r}_{i j}\right) \cdot\left(\boldsymbol{\alpha}_{j} \cdot \mathbf{r}_{i j}\right)}{r_{i j}^{2}}\right\}$.

However, in principle the Hamiltonian $H$ must be modified further to include the contributions from quantum electrodynamical corrections arising from vacuum fluctuation and vacuum polarisation. As a contribution to the total energy of the atom all these effects are calculated in first order perturbation theory.
To obtain a solution of (1) for the $N$ electron system an approximation for the wave function $\Psi$ is needed. The simplest ansatz is to use a single configuration in the form of a Slater determinant which is constructed from single particle wave functions. The variation of the expression of the total energy with respect to these single particle wave functions leads to the Hartree-Fock equations which are widely used to obtain the solutions, for non-relativistic and relativistic treatments. This approach describes the electrons in the independent particle picture, i.e. the energy beyond this ansatz is not included in the self-consistent field Hartree-Fock equations. That is the reason why the correlation energy is defined as the difference between the exact total energy and the HartreeFock or Dirac-Fock total energy [9].

\section{The correlation energy}

The definition of the correlation energy is therefore given by the expression

$E_{\text {corr }}=E_{\text {exact }}-E_{H F}$,

where, in principle, $E_{\text {exact }}$ can be measured. The problems concerning this quantity and its accuracy will be discussed in Part IV.

The subtraction of the two large quantities $E_{\text {exact }}$ and $E_{H F}$ on the right hand side of (6) is reasonable because of the two following reasons: First, one obtains a small quantity which can be discussed much easier than the large value of the total energy itself. Second, this correlation energy contains just all the contributions beyond an independent particle description. Although QED effects are not single particle contributions we do include them in the quantity $E_{H F}$ because these effects are not usually taken care of (not even in principle) in the many body theories.

The purpose of this paper is to collect all contributions to the energy of the ground state of the atoms or ions which contribute to the quantity $E_{\mathrm{HF}}$, and to give a critical review of the experimental values for elements with large $Z$ and as many electrons as possible. As final result we present the correlation energies $E_{\text {corr }}$ for 2 to 20 electrons in their ground state for $Z=2$ to 25 .

The theoretical description of these correlation energies for $N$ electron atoms is one of the main problems in theoretical atomic physics. There are several approaches to calculate this quantity. Many-body perturbation theory, as developed by Brueckner and Goldstone, has been applied to atoms to obtain corrections for the Hartree-Fock wave functions and energies. Such calculations for a wide variety of properties of neutral beryllium were first made by Kelly [10]. 
Similar calculations have been extended to simple atomic systems, essentially of the first row atoms using a number of various approximations [11-13].

A $Z$-dependent perturbation theory of many-electron atoms is another approach [14-16]. Here the total energy as well as the total wave functions are expanded in inverse powers of $Z$. In this way it becomes possible to study the $Z$-dependence of the correlation energy. Relativistic extension of $Z^{-1}$ expansion formalism was carried out first by Layzer and Bahcall [17].

A third approach to the calculation of correlation energy is the multi-configuration Hartree (Dirac)Fock approximation [5]. Here the wave function $\Psi$ in (1) is assumed to be a sum of configurations each of which are sums of Slater determinants. However, also the multi-configuration calculations grow difficult and cumbersome when applied to an increasing number of electrons in open shells. How well the ground state can be described in the multi-configuration approximation depends on the number of configuration state wave functions in the expansion of the total ground state wave function. For example, in the case of the ground state of the He-like systems the expansion into the configuration $1 s^{2}+2 s^{2}+2 p^{2}$ $+3 s^{2}+3 p^{2}+3 d^{2}$ provides already $95 \%$ of the correlation energy [18]. This can be compared with the most accurate calculation of neutral $\mathrm{He}$ by Bunge [19] who used a full CI calculation. One further method, which might be of interest in the future, is the g-Hartree approach by Dietz et al. [20], which is a relativistic method and includes QED effects as well.

However, all these methods have their limitations, because the degree of complexity to obtain results for an increasing number of electrons increases beyond practical means. Due to this complexity, independent of which method is used, the simplest many electron atoms, the 2 electron He-like and the 4 electron Be-like systems, are among the most intensively investigated. A summary of this field plus additional references can be found in the book by Lindgren and Morrison [13].

In any case, before trying to calculate such a complex quantity it is necessary to know how large the correlation energy should be. Apart from the problem of collecting all parts which contribute to the value $E_{H F}$, good experimental values for $E_{\text {exact }}$ are necessary. As these values contain errors we use the expression $E_{\text {exp }}$ instead. This energy is the sum of the ionisation potentials of the atoms or ions. Let $I P(N)$ be the ionisation potential of the $N$ electron to the $N-1$ electron groundstate system the energy $E_{\exp }$ is given by

$E_{\mathrm{exp}}(N)=\sum_{N} I P(N)$
The ionisation potentials $I P$ are measured quantities which can be taken from the Grotrian Tables [21]. They have been derived from extensive analyses of experimental spectra. We use these values for the elements from $Z=2$ to $Z=18$ [21] in order to be consistent over a wide range of elements, and because these values seem to be the best available at present. Probably they are more reliable than those given in the Tables by Moore [22] or Cowan [23]. The experimental errors for the ionisation potentials grow very large already for all elements beyond $Z=18$. Again, to be consistent, we used the most recent values for the elements from $Z=19$ to 25 from the compilation of Corliss and Sugar [24].

Values for correlation energies were first obtained on similar lines by Clementi [7] who used non-relativistic Hartree-Fock energies wih relativistic corrections as theoretical values in (6) and the ionisation potentials from the tables of Moore [22] for constructing the experimental energies.

\section{Results and discussion}

The computed Dirac-Fock values $E_{D F}$ used here already include the relativistic effects as well as the contribution from the magnetic energy, retardation and the QED effects, i.e. self-energy and vacuum polarisation as well as the effect of the extended nucleus as pointed out in Chap. 2.

In Table 1 we present the calculated Dirac-Fock energies $E_{D F}$ and the experimental total energies $E_{\text {exp }}$ as obtained from the Grotrian Tables [21] for the elements $Z=2-18$ and from [24] for the elements $Z=19$ to 25 for all isoelectronic series from 2 to 20 electrons. In addition, the differences of both energies, the correlation energy, is listed. We did not continue this listing for elements above $Z=25$ because the experimental error bars grow so large for all heavier elements that the resulting correlation energies are far from being physically realistic. We hope that in the near future further data will become available permitting extension of the discussion to larger $Z$. This is of special interest because above $Z=20$ all additional effects in addition to a normal Dirac-Fock calculation start to be important. Table 2 gives an example of the magnitude of all contributions for the elements $20,40,60$ and 80 for the 10 electron groundstate.

The computed Dirac-Fock values themselves are expected to be accurate to within a few fractions of an $\mathrm{eV}$. However, it is problematic to give a general estimate of the accuracy of the experimental values for the total energies because these are obtained by summing individual ionisation potentials. The Grotrian Tables give no explicit value for the experimen- 
Table 1. Experimental and calculated Hartree-Fock total energies for atomic iso-electronic series for $2-20$ electrons for $Z=2$ to 25 in eV. The experimental values are given with their errors [24]. The difference given in this table is the correlation energy

\begin{tabular}{|c|c|c|c|c|c|c|}
\hline$Z$ & Exp. & Calc. & Diff. & Exp. & Calc. & Diff. \\
\hline & \multicolumn{3}{|c|}{2 electrons $1 s^{2}{ }^{1} S_{0}$} & \multicolumn{3}{|c|}{3 electrons $2 s^{1}{ }^{2} S_{1 / 2}$} \\
\hline 2 & 79.00 & 77.87 & -1.13 & & & \\
\hline 3 & $-\quad 198.09$ & $-\quad 196.93$ & -1.16 & $-\quad 203.48$ & $-\quad 202.27$ & -1.21 \\
\hline 4 & $-\quad 371.61$ & $-\quad 370.43$ & -1.18 & $-\quad 389.82$ & $-\quad 388.57$ & -1.25 \\
\hline 5 & $-\quad 599.59$ & - $\quad 598.42$ & -1.17 & $-\quad 637.52$ & $-\quad 636.24$ & -1.28 \\
\hline 6 & $-\quad 882.06$ & $-\quad 880.90$ & -1.16 & -946.55 & -945.28 & -1.27 \\
\hline 7 & -1219.09 & -1217.93 & -1.16 & -1316.95 & -1315.70 & -1.25 \\
\hline 8 & -1610.71 & -1609.56 & -1.15 & -1748.82 & -1747.55 & -1.27 \\
\hline 9 & -2056.96 & -2055.84 & -1.12 & -2242.14 & -2240.89 & -1.25 \\
\hline 10 & -2557.96 & -2556.84 & -1.12 & -2797.05 & -2795.79 & -1.26 \\
\hline 11 & -3113.75 & -3112.63 & -1.12 & -3413.62 & -3412.35 & -1.27 \\
\hline 12 & -3724.41 & -3723.30 & -1.11 & -4091.94 & -4090.65 & -1.29 \\
\hline 13 & -4390.06 & -4388.94 & -1.12 & -4832.13 & -4830.81 & -1.32 \\
\hline 14 & -5110.78 & -5109.66 & -1.12 & -5634.28 & -5632.94 & -1.34 \\
\hline 15 & -5886.70 & -5885.56 & -1.14 & -6498.55 & -6497.17 & -1.38 \\
\hline 16 & -6717.94 & -6716.78 & -1.16 & -7425.08 & -7423.64 & -1.44 \\
\hline 17 & -7604.62 & -7603.45 & -1.17 & -8414.01 & -8412.51 & -1.50 \\
\hline 18 & -8546.84 & -8545.70 & -1.14 & $-9464.8[10]$ & -9463.94 & -1.0 \\
\hline 19 & $-9544.9 \quad[9]$ & -9543.71 & -1.19 & $-10578.3 \quad[9]$ & -10578.12 & -0.2 \\
\hline 20 & $-10598.7[10]$ & -10597.62 & -1.1 & $-11756.5[10]$ & -11755.22 & -1.3 \\
\hline 21 & $-11708.6[10]$ & -11707.58 & -1.0 & $-12996.6[11]$ & -12995.42 & -1.2 \\
\hline 22 & $-12874.9[10]$ & -12873.78 & -1.1 & $-14300.3[11]$ & -14298.92 & -1.4 \\
\hline 23 & $-14097.5[10]$ & -14096.42 & -1.1 & $-15667.1[10]$ & -15665.96 & -1.1 \\
\hline 24 & $-15376.7[6]$ & -15375.69 & -1.0 & $-17098.1[7]$ & -17096.76 & -1.3 \\
\hline \multirow[t]{2}{*}{25} & $-16712.7[20]$ & -16711.80 & -0.9 & $-18592.6[20]$ & -18591.57 & -1.0 \\
\hline & \multicolumn{3}{|c|}{4 electrons $2 s^{2}{ }^{1} S_{0}$} & \multicolumn{3}{|c|}{5 electrons $2 p^{12} P_{1 / 2}$} \\
\hline 4 & $-\quad 399.14$ & $-\quad 396.61$ & -2.53 & & & \\
\hline 5 & $-\quad 662.67$ & $-\quad 659.69$ & -2.98 & $-\quad 670.97$ & $-\quad 667.62$ & -3.35 \\
\hline 6 & $-\quad 994.44$ & $-\quad 991.08$ & -3.36 & -1018.82 & -1015.13 & -3.69 \\
\hline 7 & -1394.42 & -1390.72 & -3.70 & -1441.85 & -1437.90 & -3.95 \\
\hline 8 & -1862.72 & -1858.65 & -4.07 & -1940.13 & -1935.87 & -4.26 \\
\hline 9 & -2399.30 & -2394.91 & -4.39 & -2513.54 & -2509.07 & -4.47 \\
\hline 10 & -3004.32 & -2999.59 & -4.73 & -3162.25 & -3157.54 & -4.71 \\
\hline 11 & -3677.78 & -3672.78 & -5.00 & -3886.25 & -3881.39 & -4.86 \\
\hline 12 & -4419.89 & -4414.58 & -5.31 & -4685.79 & -4680.72 & -5.07 \\
\hline 13 & -5230.70 & -5225.11 & -5.59 & -5560.91 & -5555.65 & -5.26 \\
\hline 14 & -6110.34 & -6104.51 & -5.83 & -6511.77 & -6506.33 & -5.44 \\
\hline 15 & -7058.96 & -7052.92 & -6.04 & -7538.53 & -7532.92 & -5.61 \\
\hline 16 & -8076.71 & -8070.49 & -6.22 & -8641.36 & -8635.58 & -5.78 \\
\hline 17 & -9163.75 & -9157.41 & -6.34 & -9820.44 & -9814.51 & -5.93 \\
\hline 18 & $-10319.6[10]$ & -10313.85 & -5.7 & $-11075.3[10]$ & -11069.92 & -5.4 \\
\hline 19 & $-11546.3[22]$ & -11540.01 & -6.3 & $-12407.4[28]$ & -12402.01 & -5.4 \\
\hline 20 & $-12843.5[22]$ & -12836.11 & -7.4 & $-13817.5[30]$ & -13811.03 & -6.5 \\
\hline 21 & $-14209.6[23]$ & -14202.35 & -7.2 & $-15303.6[30]$ & -15297.20 & -6.4 \\
\hline 22 & $-15646.3[32]$ & -15638.95 & -7.3 &,$-16867.3[38]$ & -16860.76 & -6.5 \\
\hline 23 & $-17153.1[32]$ & -17146.18 & -6.9 & -18508.1 & -18502.01 & -6.1 \\
\hline 24 & $-18732.1[31]$ & -18724.28 & -7.8 & -20228.1 & -20221.24 & -6.9 \\
\hline \multirow[t]{2}{*}{25} & $-20380.6[45]$ & -20373.55 & -7.0 & $-22024.6[54]$ & -22018.73 & -5.9 \\
\hline & \multicolumn{3}{|l|}{6 electrons $2 p^{2}{ }^{3} P_{0}$} & \multicolumn{3}{|c|}{7 electrons $2 p^{3}{ }^{4} S_{3 / 2}$} \\
\hline 6 & -1030.08 & -1025.91 & -4.17 & & & \\
\hline 7 & -1471.45 & -1467.07 & -4.38 & -1485.98 & -1481.00 & -4.98 \\
\hline 8 & -1995.06 & -1990.43 & -4.63 & -2030.18 & -2025.02 & -5.16 \\
\hline 9 & -2600.68 & -2595.93 & -4.75 & -2663.38 & -2658.16 & -5.22 \\
\hline 10 & -3288.46 & -3283.59 & -4.87 & -3385.57 & -3380.37 & -5.20 \\
\hline 11 & -4058.40 & -4053.48 & -4.92 & -4196.79 & -4191.68 & -5.11 \\
\hline 12 & -4910.73 & -4905.70 & -5.03 & -5097.23 & -5092.15 & -5.08 \\
\hline 13 & -5845.50 & -5840.37 & -5.13 & -6086.93 & -6081.90 & -5.03 \\
\hline 14 & -6862.87 & -6857.64 & -5.23 & -7166.04 & -7161.05 & -4.99 \\
\hline
\end{tabular}


Table 1 (continued)

\begin{tabular}{|c|c|c|c|c|c|c|}
\hline$Z$ & Exp. & Calc. & Diff. & Exp. & Calc. & Diff. \\
\hline 15 & -7963.03 & -7957.66 & -5.37 & -8334.76 & -8329.75 & -5.01 \\
\hline 16 & -9146.14 & -9140.63 & -5.51 & -9593.23 & -9588.18 & -5.05 \\
\hline 17 & -10412.41 & -10406.75 & -5.66 & -10941.67 & -10936.53 & -5.14 \\
\hline 18 & $-11761.4[10]$ & -11756.22 & -5.2 & $-12379.7[10]$ & -12375.01 & -4.7 \\
\hline 19 & $-13194.0[32]$ & -13189.29 & -4.7 & $-13908.6[35]$ & -13903.84 & -4.8 \\
\hline 20 & $-14712.0[35]$ & -14706.21 & -5.8 & $-15529.6[39]$ & -15523.28 & -6.3 \\
\hline 21 & $-16312.6[36]$ & -16307.24 & -5.4 & $-17240.1[41]$ & -17233.56 & -6.5 \\
\hline 22 & $-17998.3[43]$ & -17992.65 & -5.6 & $-19042.3[47]$ & -19034.96 & -7.3 \\
\hline 23 & $-19768.1 \quad[48]$ & -19762.74 & -5.4 & $-20936.1[52]$ & -20927.80 & -8.3 \\
\hline 24 & $-21624.1[52]$ & -21617.86 & -6.2 & $-22923.1[60]$ & -22912.38 & -10.7 \\
\hline \multirow[t]{2}{*}{25} & $-23563.6[62]$ & -23558.32 & -5.3 & $-25000.6[69]$ & -24989.07 & -11.5 \\
\hline & \multicolumn{3}{|l|}{8 electrons $2 p^{4}{ }^{3} P_{2}$} & \multicolumn{3}{|c|}{9 electrons $2 p^{5}{ }^{2} P_{3 / 2}$} \\
\hline 8 & -2043.80 & -2036.91 & -6.89 & & & \\
\hline 9 & -2698.35 & -2691.44 & -6.91 & -2715.78 & -2707.14 & -8.64 \\
\hline 10 & -3449.02 & -3442.11 & -6.91 & -3489.98 & -3481.42 & -8.56 \\
\hline 11 & -4295.70 & -4288.84 & -6.86 & -4367.34 & -4358.83 & -8.51 \\
\hline 12 & -5238.49 & -5231.65 & -6.84 & -5347.73 & -5339.29 & -8.44 \\
\hline 13 & -6277.40 & -6270.63 & -6.77 & -6431.22 & -6422.84 & -8.28 \\
\hline 14 & -7412.56 & -7405.90 & -6.66 & -7617.61 & -7609.57 & -8.04 \\
\hline 15 & -8644.17 & -8637.62 & -6.55 & -8907.39 & -8899.61 & -7.78 \\
\hline 16 & -9972.33 & -9965.94 & -6.39 & -10300.56 & -10293.11 & -7.45 \\
\hline 17 & -11397.29 & -11391.08 & -6.21 & -11797.34 & -11790.28 & -7.06 \\
\hline 18 & -12918.6 [10] & -12913.24 & -5.4 & $-13397.3[10]$ & -13391.32 & -6.0 \\
\hline 19 & $-14538.0[37]$ & -14532.66 & -5.3 & $-15102.7[38]$ & -15.096 .47 & -6.2 \\
\hline 20 & $-16256.2[41]$ & -16249.60 & -6.6 & $-16913.4[43]$ & -16905.98 & -7.4 \\
\hline 21 & -18070.9 [44] & -18064.32 & -6.6 & $-18827.6[47]$ & -18820.11 & -7.5 \\
\hline 22 & $-19984.2[51]$ & -19977.09 & -7.1 & $-20847.3[54]$ & -20839.12 & -8.2 \\
\hline 23 & $-21996.1[56]$ & -21988.22 & -7.9 & $-22972.1[59]$ & -22963.33 & -8.8 \\
\hline 24 & $-24108.1[64]$ & -24098.04 & -10.1 & $-25205.1[67]$ & -25193.07 & -12.0 \\
\hline \multirow[t]{2}{*}{25} & $-26317.6[75]$ & -26306.89 & -10.7 & $-27541.6[79]$ & -27528.68 & -12.9 \\
\hline & \multicolumn{3}{|l|}{10 electrons $2 p^{6}{ }^{1} S_{0}$} & \multicolumn{3}{|c|}{11 electrons $3 s^{12} S_{1 / 2}$} \\
\hline 10 & -3511.54 & -3501.21 & -10.33 & & & \\
\hline 11 & -4414.62 & -4404.45 & -10.17 & -4419.76 & -4409.40 & -10.36 \\
\hline 12 & -5427.88 & -5417.82 & -10.06 & -5442.91 & -5432.51 & -10.40 \\
\hline 13 & -6551.21 & -6541.24 & -9.97 & -6579.66 & -6569.33 & -10.33 \\
\hline 14 & -7784.38 & -7774.76 & -9.62 & -7829.52 & -7819.49 & -10.03 \\
\hline 15 & -9127.82 & -9118.46 & -9.36 & -9192.85 & -9183.04 & -9.81 \\
\hline 16 & -10581.49 & -10572.50 & -8.99 & -10669.54 & -10660.07 & -9.47 \\
\hline 17 & -12145.62 & -12137.04 & -8.58 & -12259.81 & -12250.74 & -9.07 \\
\hline 18 & $-13819.7[10]$ & -13812.29 & -7.4 & $-13963.2[10]$ & -13955.2 & -8.0 \\
\hline 19 & $-15606.5[39]$ & -15598.47 & -8.0 & $-15782.3[39]$ & -1577376 & -8.5 \\
\hline 20 & $-17505.3[43]$ & -17495.84 & -9.4 & $-17716.6[43]$ & -17706.58 & -10.0 \\
\hline 21 & $-19514.9[47]$ & -19504.67 & -10.2 & $-19764.8[47]$ & -19753.95 & -10.8 \\
\hline 22 & $-21635.1[54]$ & -21625.19 & -9.9 & $-21926.6[54]$ & -21916.13 & -10.5 \\
\hline 23 & $-23868.1[59]$ & -23857.74 & -10.4 & $-24204.4[59]$ & -24193.45 & -10.9 \\
\hline 24 & $-26215.7[67]$ & -26202.65 & -13.0 & $-26599.8[67]$ & -26586.25 & -13.5 \\
\hline \multirow[t]{2}{*}{25} & $-28676.3[79]$ & -28660.24 & -16.1 & $-29111.6[79]$ & -29094.85 & -16.6 \\
\hline & \multicolumn{3}{|l|}{12 electrons $3 s^{2}{ }^{1} S_{0}$} & \multicolumn{3}{|c|}{13 electrons $3 p^{12} P_{1 / 2}$} \\
\hline 12 & -5450.56 & -5439.18 & -11.38 & & & \\
\hline 13 & -6598.49 & -6586.88 & -11.61 & -6604.47 & -6592.38 & -12.09 \\
\hline 14 & -7863.01 & -7851.51 & -11.50 & -7879.35 & -7867.23 & -12.12 \\
\hline 15 & -9244.22 & -9232.84 & -11.38 & -9274.40 & -9262.31 & -12.09 \\
\hline 16 & -10742.22 & -10730.87 & -11.35 & -10789.52 & -10777.42 & -12.10 \\
\hline 17 & -12356.84 & -12345.70 & -11.14 & -12424.64 & -12412.55 & -12.09 \\
\hline 18 & $-14087.5[10]$ & -14077.50 & -10.0 & $-14178.5[10]$ & -14167.83 & -10.7 \\
\hline 19 & $-15937.2[39]$ & -15926.46 & -10.7 & $-16054.7[39]$ & -16043.43 & -11.3 \\
\hline 20 & $-17905.1[43]$ & -17892.83 & -12.3 & $-18052.4[43]$ & -18039.57 & -12.8 \\
\hline 21 & $-19990.0[47]$ & -19976.85 & -13.1 & $-20170.0[47]$ & -20156.48 & -13.5 \\
\hline
\end{tabular}


Table 1 (continued)

\begin{tabular}{|c|c|c|c|c|c|c|}
\hline$Z$ & Exp. & Calc. & Diff. & Exp. & Calc. & Diff. \\
\hline 22 & $-22191.7[54]$ & -22178.80 & -12.9 & $-22407.6[54]$ & -22394.44 & -13.2 \\
\hline 23 & $-24512.5[59]$ & -24499.01 & -13.5 & $-24768.2[59]$ & -24753.78 & -14.4 \\
\hline 24 & $-26954.6[67]$ & -26937.81 & -16.8 & $-27252.6[68]$ & -27234.82 & -17.8 \\
\hline \multirow[t]{2}{*}{25} & $-29514.5[79]$ & -29495.55 & -18.9 & $-29858.1[80]$ & -29837.92 & -20.2 \\
\hline & \multicolumn{3}{|c|}{14 electrons $3 p^{2}{ }^{3} P_{0}$} & \multicolumn{3}{|c|}{15 electrons $3 p^{3}{ }^{4} S_{3 / 2}$} \\
\hline 14 & -7887.51 & -7874.86 & -12.65 & & & \\
\hline 15 & -9294.12 & -9281.47 & -12.65 & -9304.61 & -9291.45 & -13.16 \\
\hline 16 & -10824.35 & -10811.56 & -12.79 & -10847.68 & -10834.34 & -13.34 \\
\hline 17 & -12478.10 & -12464.98 & -13.12 & -12517.71 & -12503.97 & -13.74 \\
\hline 18 & $-14253.9[10]$ & -14241.77 & -12.1 & $-14313.7[10]$ & -14300.23 & -13.5 \\
\hline 19 & -16154.1 [39] & -16142.04 & -12.1 & $-16236.8[39]$ & -16223.17 & -13.6 \\
\hline 20 & $-18179.6[43]$ & -18165.98 & -13.6 & $-18288.3[43]$ & -18272.92 & -15.4 \\
\hline 21 & $-20328.1[47]$ & -20313.82 & -14.3 & $-20466.1[47]$ & -20449.69 & -16.4 \\
\hline 22 & -22599.7 [54] & -22585.82 & -13.9 & $-22770.1[54]$ & -22753.69 & -16.4 \\
\hline 23 & $-24998.7[59]$ & -24982.30 & -16.4 & $-25204.5[60]$ & -25185.24 & -19.3 \\
\hline 24 & $-27523.4[68]$ & -27503.59 & -19.8 & $-27767.8[68]$ & -27744.66 & -23.1 \\
\hline \multirow[t]{2}{*}{25} & $-30172.5[80]$ & -30150.05 & -22.4 & $-30458.5[80]$ & -30432.29 & -26.2 \\
\hline & \multicolumn{3}{|c|}{16 electrons $3 p^{4}{ }^{3} P_{2}$} & \multicolumn{3}{|c|}{17 electrons $3 p^{52} p_{3 / 2}$} \\
\hline 16 & -10858.04 & -10843.33 & -14.71 & & & \\
\hline 17 & -12541.52 & -12526.29 & -15.23 & -12554.49 & -12538.14 & -16.35 \\
\hline 18 & $-14354.4[10]$ & -14339.31 & -15.1 & $-14382.1[10]$ & -14365.82 & -16.3 \\
\hline 19 & $-16297.7[39]$ & -16282.31 & -15.4 & $-16343.5[39]$ & -16326.92 & -16.6 \\
\hline 20 & $-18372.8[43]$ & -18355.35 & -17.4 & $-18440.1[43]$ & -18421.38 & -18.7 \\
\hline 21 & $-20576.8[47]$ & -20558.56 & -18.2 & $-20668.7[47]$ & -20649.26 & -19.4 \\
\hline 22 & $-22910.9[54]$ & -22892.13 & -18.8 & $-23030.4[54]$ & -23010.71 & -19.7 \\
\hline 23 & $-25377.9[60]$ & -25356.36 & -21.5 & $-25528.5[60]$ & -25506.00 & -22.5 \\
\hline 24 & $-27977.1[68]$ & -27951.55 & -25.5 & $-28161.8[68]$ & -28135.40 & -26.4 \\
\hline \multirow[t]{2}{*}{25} & $-30706.8[80]$ & -30678.02 & -28.8 & $-30928.6[80]$ & -30899.25 & -29.3 \\
\hline & \multicolumn{3}{|c|}{18 electrons $3 p^{62} S_{0}$} & \multicolumn{3}{|c|}{19 electrons $4 s^{12} S_{1 / 2}$} \\
\hline 18 & $-14397.8[10]$ & -14380.51 & -17.3 & & & \\
\hline 19 & -16375.1 [39] & -16357.45 & -17.6 & $-16379.5[39]$ & -16361.47 & -18.0 \\
\hline \multirow[t]{2}{*}{20} & $-18491.0[43]$ & -18471.15 & -19.8 & $-18502.9[43]$ & -18482.51 & -20.4 \\
\hline & & & & \multicolumn{3}{|c|}{19 electrons $3 d^{12} D_{3 / 2}$} \\
\hline 21 & $-20742.2[47]$ & -20721.56 & -20.6 & $-20766.9[47]$ & -20745.30 & -21.1 \\
\hline 22 & $-23129.7[54]$ & -23108.76 & -20.9 & $-23173.0[54]$ & -23150.95 & -22.0 \\
\hline 23 & $-25656.6[60]$ & -25632.96 & -23.6 & $-25721.9[60]$ & -25697.13 & -24.8 \\
\hline 24 & $-28322.0[68]$ & -28294.42 & -27.6 & $-28412.7[68]$ & -28383.90 & -28.8 \\
\hline \multirow[t]{2}{*}{25} & $-31123.1[80]$ & -31093.43 & -29.7 & $-31242.3[80]$ & -31211.47 & -30.8 \\
\hline & \multicolumn{6}{|c|}{20 electrons $4 s^{2} S_{0}$} \\
\hline \multirow[t]{2}{*}{20} & $-18509.0[43]$ & -18487.65 & -21.35 & & & \\
\hline & \multicolumn{6}{|c|}{20 electrons $4 s^{1} 3 d^{13} D_{1}$} \\
\hline \multirow[t]{2}{*}{21} & $-20779.7[47]$ & -20757.54 & -22.16 & & & \\
\hline & \multicolumn{6}{|c|}{20 electrons $3 d^{2}{ }^{3} F_{2}$} \\
\hline 22 & $-23200.5[54]$ & -23177.55 & -22.95 & & & \\
\hline 23 & $-25768.6[60]$ & -25743.00 & -25.60 & & & \\
\hline 24 & $-28482.1[68]$ & -28452.59 & -29.51 & & & \\
\hline 25 & $-31337.9[80]$ & -31306.35 & -31.55 & & & \\
\hline
\end{tabular}

tal error of each ionisation energy, thus an overall error is very hard to estimate. Nevertheless, for all elements below Argon the accuracy of the experimental total energy seems to be very good within less than a few tenth of an $\mathrm{eV}$ at the most for all ionisation stages. For $Z=18$ the error is already in the order of $1 \mathrm{eV}$, whereas for the elements above Argon the average experimental error can be calculated because 
Table 2. Contributions to the total energy for 10 electron systems and $Z=20,40,60$ and 80 in eV

\begin{tabular}{lrrrr}
\hline Element & \multicolumn{1}{l}{$Z=20$} & \multicolumn{1}{l}{$Z=40$} & \multicolumn{1}{l}{$Z=60$} & \multicolumn{1}{c}{$Z=80$} \\
\hline Hartree-Fock & -17425.90 & -77956.17 & -182036.90 & -329746.76 \\
Extended nucleus & 0.04 & 1.13 & 13.95 & 125.73 \\
Relativistic contr. & -78.14 & -1468.72 & -8238.41 & -29111.51 \\
Magnetic contr. & 5.40 & 52.69 & 195.00 & 501.41 \\
Retardation & -0.42 & -4.55 & -17.05 & -42.91 \\
QED-effects & 3.18 & 34.74 & 138.27 & 379.06 \\
\hline Total energy & -17495.84 & -79340.88 & -189945.14 & -357894.98 \\
\hline
\end{tabular}

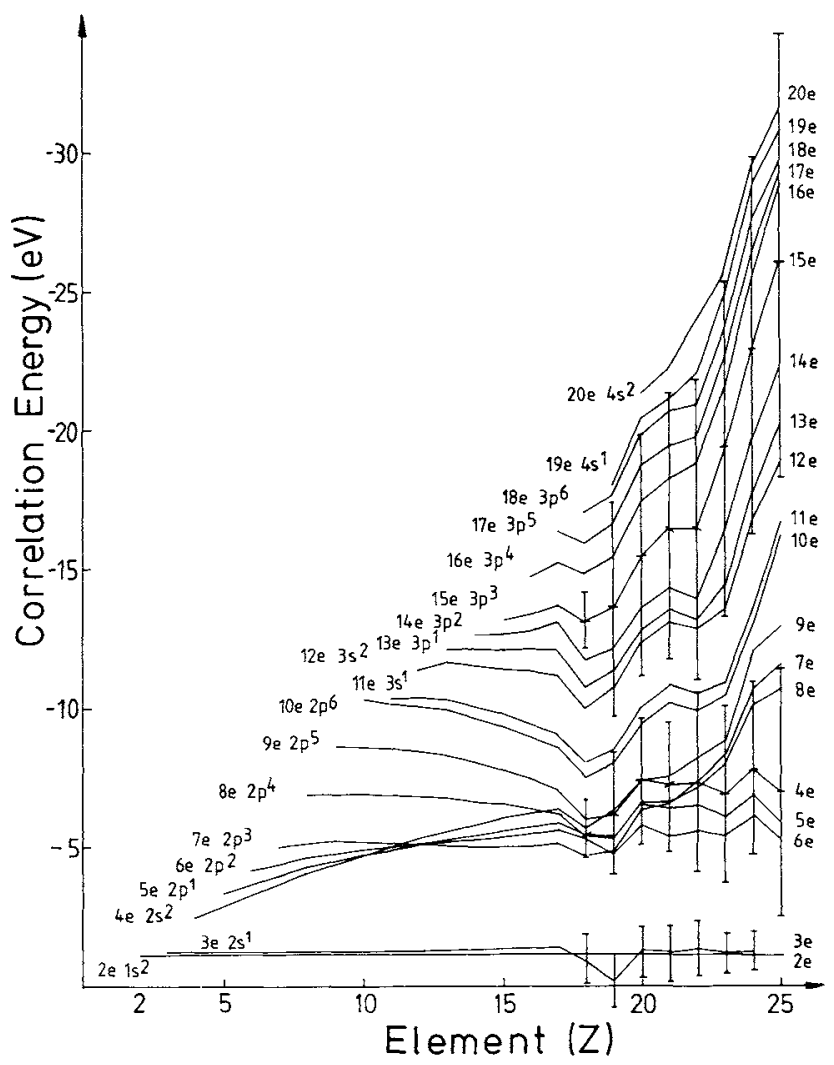

Fig. 1. Correlation energies of the isoelectronic series of 2 to 20 electrons and elements from $\mathrm{He}(Z=2)$ to $\mathrm{Mn}(Z=25)$. The error bars for the elements below Argon are below an tenth of an $\mathrm{eV}$. For all elements above $\mathrm{Cl}$ the average sum of the experimental errors from [24] are given. Because of the large values we present these errors only for the 3,4 and 15 isoelectronic series

Sugar and Corliss [24] assign an error to each ionisation potential.

The results given in Table 1 are put together in Fig. 1. The correlation energies are plotted against the nuclear charge $Z$ for all isoelectronic series of 2 to 20 electrons up to $Z=25$. At a few points error bars indicate the experimental errors. The values below $Z=18$ are so accurate that no error bars are given. The values for $Z=18$ and above have error bars which become very large. Therefore we only included them for the isoelectronic series for 3, 4 and 15 electrons. For all other series the error bars are of the same order of magnitude (see Table 1). These error bars include the full experimental errors for the total energy of the ionization stage under discussion. (Of course, the error decreases strongly if only differences between certain ionization stages are discussed.) The most striking feature in Fig. 1 is the irregular behaviour at $Z=18$ and the strong variation of the curves for the elements beyond.

In general the $E_{\text {corr }}$ curves possess both the magnitude and the systematic $Z$-dependence behaviour of the correlation energies, as known e.g. from the $Z$ expansion method. Especially the two-electron systems with almost no error bars have been reproduced theoretically with high accuracy. The correlation energies for all systems up to Ne agree absolutely with the values of Clementi's [7] previous work. His values for larger $Z$ behave comparatively regular with increasing nuclear charge $Z$. This is rather unexpected since the ionisation potentials used by Clementi are from Moores [20] Tables which are not of the required accuracy for highly ionized few-electron systems. An explanation may be that in special cases values may have been corrected or estimated by extrapolation along the isoelectronic series.

The main reason for using the experimental values of Corliss and Sugar for elements beyond Argon is that a sharp inconsistent decrease of the correlation energy would occur if, for example, the experimental values from the Grotrian Tables would have been used. The difference of only a few eV or even parts of it are made obvious in Fig. 1. Although we do not overestimate the accuracy of the theoretical values the consistency or inconsistency of the values in Fig. 1 leads us to believe the quality of the theoretical values to be much better for $Z$ above 18 than the experimental information available.

It is of interest to compare trends for the same electronic shell with different main quantum numbers. Figure 2 shows the relative correlation energy for the 


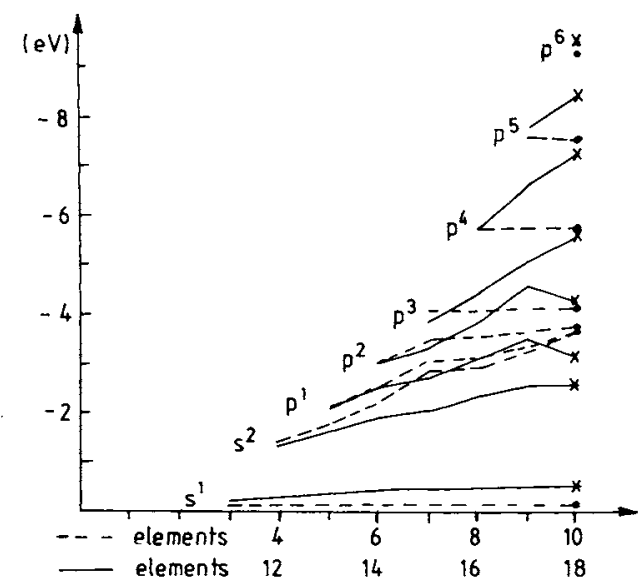

Fig. 2. Differences of correlation energies for the $2 s, 2 p$ and $3 s, 3 p$ elements. For the elements $\mathrm{Li}$ to $\mathrm{Ne}$ the values of the correlation energy of the 2 electron systems and for the elements $\mathrm{Mg}$ to $\mathrm{Ar}$ the values of the 10 electron systems are subtracted

$2 s, 2 p$ and the $3 s, 3 p$ elements where we have subtracted the value of the correlation energy of the 2 electron system for the $2 s$ and $2 p$ elements (dashed lines) and the correlation energy of the 10 electron system from the values for the $3 s$ and $3 p$ elements (full lines). It also shows that the relative correlation energy starts at almost the same value for all neutral systems however deviates for higher ionisation. Obviously this is an indirect effect of the electrons of the core in the $3 p$ elements. This also shows that a perturbation expansion for the higher elements probably will be very difficult.

\section{Summary}

Due to the problems's complexity, both theoretically and experimentally, good correlation energies are known only for a very small number of elements and few isoelectronic series. We updated and expanded the values for this important quantity correlation energy to $Z=25$ and 2 to 20 electrons. In order to understand the absolute values of the total energies for even higher $Z$ more sophisticated theoretical and experimental work has to be done. This is not only a matter of available computer power but also of a much better understanding of basic physical questions.

We would like to thank Dr. J.-P. Desclaux for a copy of this newest version of his multi-configuration Dirac-Fock program. Two of us (K.R. and B.F.) are greatful to the Kernforschungszentrum Karlsruhe and Pakistan Atomic Energy Commision for financial support under the Pakistan-German collaboration program. This work was also partially supported by the Deutsche Forschungsgemeinschaft (DFG) under contract Fr 637/3-3.

\section{References}

1. Froese-Fischer, C.: Comp. Phys. Commun. 4, 107 (1972)

2. Desclaux, J.P.: Comp. Phys. Commun. 9, 31 (1975)

3. Grant, I.P., McKenzie, B.J., Norrigton, P.H., Mayers, D.F., Pyper, N.C.: Comp. Phys. Commun. 21, 207 (1980)

4. Munger, C., Gould, H.: Bull. Am. Phys. Soc. 30, 860 (1985)

5. Fischer, C.F.: The Hartree-Fock method for atoms. New York: John Wiley \& Sons 1977

6. Johnson, W.R., Soff, G.: Atom. Data Nucl. Data Tables 33, 405 (1985)

7. Clementi, E.: J. Chem. Phys. 38, 2248 (1963); 39, 175 (1963); 42, $2783(1965)$

8. A general discussion with numerical results of the various approximations of the Breit operator can be found in: Mann, J., Johnson, W.R.: Phys. Rev. A 4, 41 (1971)

9. Löwdin, P.O.: Adv. Chem. Phys. 2, 207 (1959)

10. Kelly, H.P.: Phys. Rev. 131, 684 (1963); 136 B, 896 (1965)

11. Nesbet, R.K.: Phys. Rev, 155, 51 (1967)

12. Lindgren, I., Salomonson, S.: Phys. Scr. 21, 355 (1980)

13. Lindgren, I., Morrison, J.: Atomic many-body theory. (Springer Series in Chem. Physics 13) Berin, Heidelberg, New York: Springer 1982

14. Layzer, D., Horak, Z., Lewis, M.N., Thompson, D.P.: Ann. Phys. 29, 101 (1964); Layzer, D.: Ann. Phys. 8, 271 (1959)

15. Hibbert, A.: Rep. Prog. Phys. 38, 1217 (1975)

16. e.g. Sofranova, U.I.: Phys. Scr. 23, 241 (1981)

17. Layzer, D., Bahcall, J.: Ann. Phys. 17, 177 (1962)

18. Froese-Fischer, C.: J. Comp. Phys. 13, 502 (1973)

19. Bunge, C.F.: Theor. Chim. Acta Berlin 16, 126 (1970)

20. Dietz, K., Weymans, G.: J. Phys. B 17, 4801 (1984); Physica 131 A, 363 (1985)

21. Bashkin, S., Stoner, P.: Atomic energy levels and grotrian tables. Amsterdam: North Holland, 1975

22. Moore, C.E.: Atomic energy levels NSRDS-NBS-35. Washington D.C.: U.S. Gov. Printing Office 1971

23. Cowan, R.D.: The theory of atomic structure and spectra. University of California Press 1981

24. Sugar, J., Corliss, C.: J. Phys. Chem. Ref. Data 14, (Suppl. 2) 1985 